\title{
OSTWALD RIPENING IN POROUS MATERIALS
}

Jürn Schmelzer, Jörg Möller

Fachbereich Physik der Universität Rostock - Universitätsplatz - D-18051 - Rostock - Germany

Vitali V. Slezov

National Science Center - Kharkov Institute of Physics and Technology - Academician Street 1 - 310108 Kharkov - Ukraine

Iwan Gutzow, R. Pascova

Institute of Physical Chemistry - Bulgarian Academy of Sciences - Sofia 1113 - Bulgaria

Recebido em 2/12/96; aceito em 29/9/97

\begin{abstract}
The process of coarsening of an ensemble of clusters is investigated for the case that elastic strains due to matrix - cluster interactions change the process qualitatively as compared with dependencies established theoretically first by Lifshitz and Slezov. Such a qualitatively different behavior occurs always when the energy of elastic deformation in cluster growth increases more rapidly than linear with the volume of a cluster. Analytic solutions, for limiting cases, as well as numerical solutions, for the general case of coarsening in an ensemble of pores with a given pore size distribution, are presented. Possible applications are discussed.
\end{abstract}

Keywords: phase transformations in solids; elastic strains; coarsening.

\section{INTRODUCTION}

The present work was originated by attempts to explain theoretically results of Gutzow and Pascova ${ }^{1}$ on coarsening of a segregating silver chloride phase in highly viscous glassforming melts. In these experiments, after an initial period of coarsening of an ensemble of clusters, described by wellknown dependencies established first by Lifshitz and Slezov ${ }^{2}$

$\langle R\rangle^{3} \alpha t, \mathrm{~N} \alpha t^{-1}$

a sudden switch to a stage was observed, where cluster number, $N$, and average cluster size, $\langle R\rangle$, remained practically constant for extended periods of time, $t$.

In order to understand such kind of behavior, the possible effect of elastic strains on cluster growth and coarsening was considered. However in following such line of thinking, difficulties occured. In one of the first investigations of Lifshitz and Slezov on the effect of elastic strains on coarsening the conclusion was drawn that elastic stresses may lead only to quantitative modi-fications but not to a qualitative change of the basic features of the process.

Therefore, the problem was to analyse under which conditions such conclusion is true and, vice versa, under which conditions may be not valid. For this purpose, different models of evolution of elastic strains were analyzed as a first step.

\section{MODELS OF ELASTIC STRAINS IN CLUSTER GROWTH}

The most widely employed model in considering elastic strains in phase transformations in solids is directed to the analysis of elastic strains resulting from a misfit between ambient and newly evolving solid phases ${ }^{3,4}$. The same kind of strains is also of considerable importance for the understanding of crystallization processes in glasses, in particular, the preferential surface crystallization in systems where the differences between volume per particle in the glass and the crystalline phase are particularly high ${ }^{5}$.

Provided, in the bulk of a solid, a cluster of a new phase is formed, the total energy of elastic deformations, $\Phi^{(\varepsilon)}$, connected with the transformation may be expressed as

$\Phi^{(\varepsilon)}=\varepsilon V$
The parameter $\varepsilon$ is determined by the elastic constants of both phases (Young's modulus, $E$, and Poisson's number, $\gamma$ ), $V$ is the volume of the cluster.

The change of the Gibbs free energy $\Delta G$ in cluster formation gets then the form

$\Delta G=-n \Delta \mu+\sigma A+\varepsilon V$

or

$\Delta G=-n\left(\Delta \mu-\frac{\varepsilon}{c_{\text {cluster }}}\right)+\sigma A$

Here $n$ is the number of particles in the cluster, $c_{\text {cluster }}$ their volume concentration, $\Delta \mu$ is the difference in the chemical potencial per particle between ambient and newly evolving phases, respectively, $\sigma$ the specific interfacial energy and $A$ the surface area of a cluster.

An inspection of equation (4) shows that the effect of elastic strains on cluster growth for this model of evolution of strains does not depend on the size of the cluster. Therefore, strains of the type considered so far (and analyzed originally also by Lifshitz and Slezov) cannot affect the coarsening behavior qualitatively.

The situation may become, however, quite different if elastic strains in segregation processes in multicomponent solu tions are considered. Suppose, one of the components of a binary solution segregates and has a diffusion coefficient $D$ considerably larger as compared with the ambient phase particles. In this case, elastic strains in segregation evolve resembling the deformation behavior of an elastic spring. For elastically deformed springs, the force is proportional to the elongation, the energy is proportional to the elongation squared.

If the initial volume of a cluster, when such type of strains begin to act, is denoted as $V_{\mathrm{o}}$, the elastic strains in cluster growth can be written in this case as

$\Phi^{(\varepsilon)}=\kappa \frac{\left(V-V_{o}\right)^{2}}{V_{o}} \Theta$, 
$\Theta= \begin{cases}1 & \text { for } V-V_{o}>0 \\ 0 & \text { for } V-V_{o} \leq 0\end{cases}$

The parameter $\kappa$ is some combination of elastic constants, again.

The change of the Gibbs free energy in cluster formation can be written in this case as

$\Delta G=-n \Delta \mu+\sigma A+\kappa \frac{\left(V-V_{o}\right)^{2}}{V_{o}} \Theta$

or

$\Delta G=-n\left[\Delta \mu-\frac{\kappa}{c_{\text {cluster }}}\left(\frac{\left(V-V_{o}\right)^{2}}{V V_{o}}\right) \Theta\right]+\sigma A$

For this model, the influence of elastic strains increases with increasing cluster size and may, consequently, also qualitatively change the coarsening behavior. Therefore, the problem arises to develop a theory of cluster growth and coarsening under the influence of such qualitatively different, non-linear in the cluster volume, types of elastic strains.

\section{COARSENING AT NON-LINEAR INCREASE OF THE ENERGY OF ELASTIC STRAINS: A FIRST APPROACH}

The first approach to the description of coarsening under the influence of elastic strains was based on a thermodynamic analysis of the process of first-order phase transformations ${ }^{6}$. It results in the derivation of differential equations describing the evolution of the average cluster size, $\langle R\rangle$, and the number of clusters, $N$, in the system (see Schmelzer (1985) ${ }^{7}$; Schmelzer, Gutzow(1988) ${ }^{8}$; Schmelzer, Gutzow Pascova ${ }^{9,10}$; Gutzow, Schmelzer $(1995)^{11}$. The respective equations read

$\frac{d\langle R\rangle}{d t}=\frac{8 D c}{27 c_{\text {cluster }}^{2} k_{B} T} \frac{1}{\langle R\rangle^{2}}\left\{\sigma+\frac{3}{4 \pi\langle R\rangle^{2}}\left[\Phi^{(\varepsilon)}-V \frac{\partial \Phi^{(\varepsilon)}}{\partial V}\right]\right\}$.

$\frac{1}{Z}\left\{1+Z-\frac{\langle R\rangle^{2}}{2 \sigma} \frac{\partial^{2} \Phi^{(\varepsilon)}}{\partial\langle R\rangle \partial V}\right\}$

$\frac{d \ln (N\langle n\rangle)}{d t}=-\frac{1}{Z}\left\{\left[1-\frac{\langle R\rangle^{2}}{2 \sigma} \frac{\partial^{2} \Phi^{(\varepsilon)}}{\partial\langle R\rangle \partial V}\right] \frac{d}{d t}\left[\ln \langle R\rangle^{3}\right]\right\}$.

Here $c$ is the actual concentration of segregating particles in the ambient phase, $D$ their diffusion coefficient, $k_{B}$ Boltzmann's constant and $T$ the absolute temperature, $\langle n\rangle$ the number of segregating particles in a cluster of size $\langle R\rangle$. The quantity $Z$ reflects specific properties of the system under consideration. Quite generally, the relation $Z<-1$ holds. The absolute value of this parameter increases rapidly in the course of the coarsening process.

Above equations allow to describe the whole coarsening process including its initial stages. With respect to the influence of elastic strains, the following consequences can be drawn from these results:

- If $\Phi^{(\varepsilon)}=0$ (absence of elastic strains) or $\Phi^{(\varepsilon)}=\varepsilon V$, elastic strains do not modify the coarsening process qualitatively. The LifshitzSlezov results for $\langle R(t)\rangle$ and $N(t)$ are obtained as special cases.

- If elastic strains result in energies of elastic deformations growing more rapidly than linear with the cluster volume, a qualitative change of the coarsening behavior occurs.
Note that the mechanism of inhibition of cluster growth discussed here is due to cluster - matrix interactions. Later an alternative mechanism of inhibition of coarsening was developed by Kawasaki and Enomoto ${ }^{12}$. This mechanism, not considered here, is due to elastic field interactions of different clusters.

The theoretical results obtained allow to give a satisfactory explanation of the experiments of Pascova and Gutzow and similar dependencies obtained later. It has additional advantage connected with its simplicity and straightforward applicability.

The theory is however limited in its scope, it gives only expressions for the average cluster size and the number of clusters in the system. Thus, a detailed study of coarsening processes (description of the evolution of the cluster size distribution function and related quantities), when clustermatrix interactions result in deformation energies growing more rapidly than linear with the volume of a cluster, is of interest. The results may be of importance for the understanding of coarsening in porous materials (vycor glasses, zeolithes, etc) or segregation in higly viscous melts and polymers.

Different approaches in this respect are summarized in the subsequent sections (for details see also Schmelzer, Möller (1992) $^{13}$; Slezov, Schmelzer, Möller (1993) ${ }^{14}$; Schmelzer, Möller, Slezov $\left.(1995)^{15}\right)$.

\section{OSTWALD RIPENING IN A SYSTEM OF HARD PORES OF EQUAL SIZE $R_{O}$}

We consider first the case that an ensemble of clusters evolves in a system of pores of equal size $R_{\mathrm{o}}$. The matrix is absolutely rigid, i.e., the growth of the clusters is terminated immediately, once the cluster size $R$ becomes equal to $R_{\mathrm{o}}$.

The growth equation reads

$\frac{d R}{d t}=\frac{R_{c o}^{3}}{R}\left(\frac{1}{R_{c}}-\frac{1}{R}\right) \Theta\left(R_{o}-R\right)$,

$\Theta\left(R_{o}-R\right)= \begin{cases}1 & \text { for } R_{o}-R>0 \\ 0 & \text { for } R_{o}-R \leq 0\end{cases}$

The critical cluster radius $R_{c}$ is given by

$R_{c}(t)=\frac{2 \sigma}{c_{\alpha} \Delta \mu}$,

$R_{c o}$ is its initial value in coarsening. Moreover, the time scale is measured in appropriately chosen dimensionless units (cf. ${ }^{13}$ ). The evolution of the cluster size distribution, $f(R, t)$, is determined by the continuity equation

$\frac{\partial f(R, t)}{\partial t}+\frac{\partial}{\partial R}\left[f(R, t) \frac{d R}{d t}\right]=0$

The solution of the continuity equation, assuming that initially a Lifshitz-Slezov distribution, $P_{L S}$, is established in the system, reads ${ }^{14}$

$f(R, t)=\frac{N\left(t_{o}\right)}{R_{c}\left(t_{o}\right)} P_{L S}\left[\frac{R_{l}(R, t)}{R_{c}\left(t_{o}\right)}\right] \frac{\partial R_{I}}{\partial R}, \quad R<R_{o}$

Here $t_{\mathrm{o}}$ is the time when the interactions of the cluster ensemble with the walls of the pores become of importance, $R_{1}(R, t)$ is the so-called characteristic solution of the growth equation.

$\frac{d R}{d t}=\frac{R_{c o}^{3}}{R}\left(\frac{1}{R_{c}}-\frac{1}{R}\right)$ 
The distribution function for $R \geq R_{\mathrm{O}}$ is given by

$$
f(R, t)=\left(\left.\int_{t_{0}}^{t} d t f\left(R_{0}-\varepsilon\right) \frac{d R}{d t}\right|_{R_{0}-\varepsilon} \delta\left(R_{0}-R\right)\right)_{\varepsilon \rightarrow 0}
$$

$$
R \geq R_{0} \text {. }
$$

In the course of time a monodisperse distribution of clusters develops with an average cluster size $\langle R\rangle=R_{0}$.

The method is equally well applicable for any arbitrary initial distribution. The final state is the same independent of the initial conditions.

\section{OSTWALD RIPENING IN A SYSTEM OF WEAK PORES}

As a next limiting case, allowing an analytical treatment, it is assumed that the inhibiting effect of elastic strains increases only slowly with an increasing size of the cluster. For this case, the growth equation is written as ${ }^{13,15}$

$$
\frac{d R}{d t}=\frac{R_{c o}^{3}}{R}\left\{\frac{1}{R_{c}}-\frac{1}{R}\right\}-\Phi\left(R, R_{o}\right)
$$

For the analysis, reduced variables ${ }^{2}$

$$
u=\frac{R}{R_{c}(t)}, \quad x=\frac{R_{c}(t)}{R_{c o}}, \quad \tau=\ln \left[\frac{R_{c}(t)}{R_{c o}}\right]
$$

are introduced. In these variables, the growth equation reads

$$
\frac{d u^{3}}{d \tau}=\gamma(\tau)\left\{u-1-\frac{u^{2} x^{2} \Phi\left(R, R_{o}\right)}{R_{c o}}\right\}-u^{3}
$$

with

$$
\gamma(\tau)=\left\{\frac{x^{2} d x}{d t}\right\}^{-1}
$$

In the asymptotic stage of coarsening, the relations

$$
\begin{aligned}
& F(u)=\frac{\partial}{\partial u} F(u)=0 \\
& F(u)=\gamma(\tau)\left\{u-1-\frac{u^{2} x^{2} \Phi\left(R, R_{o}\right)}{R_{c o}}\right\}-u^{3}
\end{aligned}
$$

have to be fulfilled. The two solutions $\left(u_{1}\right.$ and $\left.u_{2}\right)$ for $u$ and the value of $\gamma$ are given then by

$u=\frac{3}{2}-\frac{9 x^{2} \Phi}{8 R_{c o}}\left[\frac{3 \Phi u}{2 \Phi}-1\right], \quad \Phi_{u}=\frac{\partial \Phi}{\partial u}$

$\gamma=\frac{\left\{\frac{3}{2}-\frac{9 x^{2} \Phi}{8 R_{c o}}\left[\frac{3 \Phi_{u}}{2 \Phi}\right]\right\}^{3}}{\left\{\frac{1}{2}-\frac{9 x^{2} \Phi}{8 R_{c o}}\left[\frac{3 \Phi_{u}}{2 \Phi}+1\right]\right\}}$

From above relations, we obtain, in a good approximation, the following expression for the evolution of the critical cluster size $x^{2} \frac{d x}{d t}=\frac{4}{27}\left\{1-\frac{9 x^{2} \Phi}{4 R_{c o}}\left[\frac{3 \Phi u}{2 \Phi}+1\right]\right\}$

The cluster size ditribution function can be written in the form

$\varphi(u, \tau)=N(\tau) P(u)$

$P(u)=\frac{3 u^{2} \exp \left[-\frac{3 C_{1} u}{\left(u_{2}-u\right) u_{2}}\right] u_{2}^{3 C_{2}} u_{1}^{3 C_{1}}}{\left(u_{2}-u\right)^{3 C_{2}+2}\left(u+u_{1}\right)^{3 C_{2}+1}}$

with

$C_{1}=\frac{u_{2}^{2}}{u_{1}+u_{2}}, \quad C_{2}=\frac{u_{2}\left(2 u_{1}+u_{2}\right)}{\left(u_{1}+u_{2}\right)^{2}}, \quad C_{3}=\frac{u_{1}^{2}}{\left(u_{1}+u_{2}\right)^{2}}$

In the limiting case $\Phi=0$, the Lifshitz-Slezov distribution $P_{L S}(u)$ is obtained as a special case:

$P_{L S}(u)=\frac{3^{4} e \exp \left\{-\frac{3}{[2(3 / 2-u)]}\right\}}{2^{5 / 3}(u+3)^{7 / 3}(3 / 2-u)^{11 / 3}}$

In general, the parameters $u_{1}$ and $u_{2}$ are slowly varying functions of time. This way, also the function $P(u)$ varies slowly with time. The way of variation depends hereby on the type of response of the matrix.

\section{COARSENING IN A SYSTEM OF NON DEFORMABLE PORES WITH A GIVEN PORE-SIZE DISTRIBUTION}

\subsection{A First Approximation}

Till now, the coarsening process was considered in a system of pores of equal size. In real porous materials, there exists always some given pore size distribution we denote as $W\left(R_{0},\left\langle R_{0}\right\rangle\right)$. The parameter $\left\langle R_{0}\right\rangle$ has the meaning of the average pore size. The distribution function $W$ obeys the condition

$\int_{0}^{\infty} W\left(R_{o},\left\langle R_{o}\right\rangle\right) d R_{o}=1$

In order to apply the methods developed earlier, one may introduce an effective or average growth rate of the clusters as

$\left\langle\frac{d R}{d t}\right\rangle=\int_{0}^{\infty} d R_{o}\left[W\left(R_{o},\left\langle R_{o}\right\rangle\right) \frac{d R}{d t}\right]$

or

$$
\left\langle\frac{d R}{d t}\right\rangle=\frac{R_{c o}^{3}}{R}\left(\frac{1}{R_{c}}-\frac{1}{R}\right)_{0}^{\infty} d R_{o}\left[W\left(R_{o},\left\langle R_{o}\right\rangle\right) \Theta\left(R_{o}-R\right)\right]
$$

The effective growth rate $\left\langle\frac{d R}{d t}\right\rangle$ is a function of the quantities $R, R_{c}$ and $\left\langle R_{\mathrm{o}}\right\rangle$, only. Consequently, the same methods may be applied as discussed above by identifying $R_{o}$ with $\left\langle R_{\mathrm{o}}\right\rangle$. 


\subsection{General Approach: Description of the Method}

In a more general and accurate picture, one has to introduce a probability density $w\left(R, R_{o}\right)$ describing the distribution of clusters of size $R$ in pores of size $R_{o}$. We get

$d N=w\left(R, R_{o}, t\right) d R d R_{o}, \quad f(R, t)=\int_{0}^{\infty} w\left(R, R_{0}, t\right) d R_{0}$

Assuming that initially cluster and pore size distribution are independent, i.e.

$w\left(R, R_{o}, t\right)=f(R, t) W\left(R_{o}\right)$

the further evolution of the distribution can be followed by numerical methods. Results are shown on figs. 1 and 2.

\section{INFLUENCE OF STOCHASTIC EFFECTS ON COARSENING IN POROUS MATERIALS}

The approach outlined in the preceding sections is based on the solution of deterministic equations describing the growth of an ensemble of clusters. Stochastic effects may be incorporated into the description by applying the basic set of kinetic equations underlying classical nucleation theory (see e.g. ${ }^{11}$ )

$\frac{\partial f(n, t)}{\partial t}=J(n-1, t)-J(n, t)$

$J(n, t)=w^{(+)}(n, t) f(n, t)-w^{(-)}(n+1, t) f(n+1, t)$

The coefficients of aggregation $w^{(+)}$and emission $w^{(-)}$of
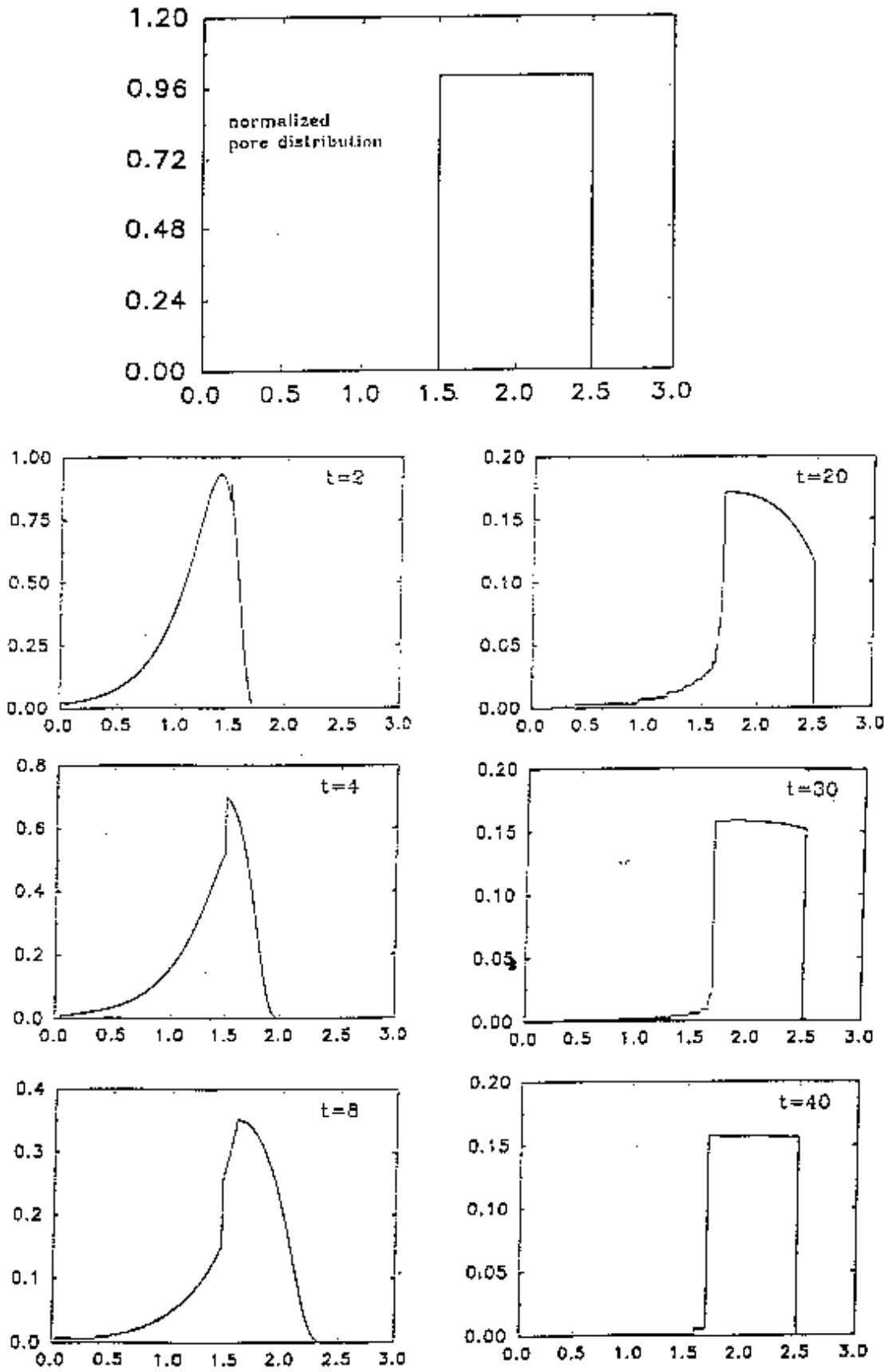

Figura 1. Different stages in the time evolution of the cluster size distribution. The pore-size distribution is shown at the top of the figure. The evolution of the cluster size distribution starts by assumption with the LS-distribution. 

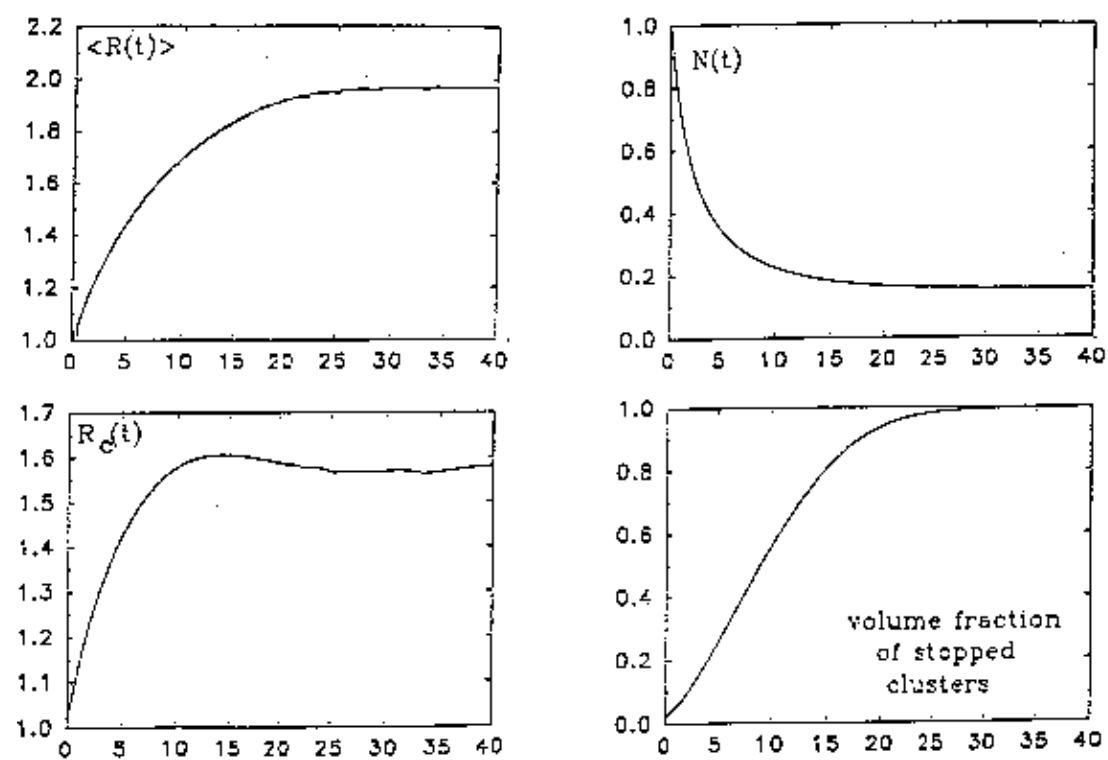

Figura 2. Time evolution of the average cluster radius $\langle R\rangle$, the critical clusters radius $R_{c}$, the number of cluster $N(t)$ in the system and the amount of the new phase immobilized in the pores as functions of time for the process shown in Fig. 1 as obtained from the numerical calculations.

particles from/to the cluster have to be determined hereby from the macroscopic growth rates and additional relations $\left(\right.$ cf. $\left.^{16}\right)$.

Provided the case of cluster growth in an ensemble of pores of equal size in an absolutely rigid matrix is considered, the stationary solution for large times is

$f(n-1)=\frac{w^{(-)}(n)}{w^{(+)}(n-1)} f(n), \quad n=j_{\max }$,

$f(n-k)=\frac{w^{(-)}(n) w^{(-)}(n-1) \ldots w^{(-)}(n-(k-1))}{w^{(+)}(n-1) w^{(+)}(n-2) \ldots w^{(+)}(n-k)} f(n)$

Taking into account stochastic effects, as one new property some broadening of the asymptotic distribution occurs, consequently.

\section{DISCUSSION}

Summarizing the results, we may conclude that theoretical approaches for the description of coarsening have been developed for the case that cluster-matrix interactions qualitative change the kinetics of coarsening as compared with the Lifshitz-Slezov theory or its modifications. We found

- analytical methods of description of coarsening for the cases of hard and weak pores of equal size;

- methods of numerical solution for the general case of coarsening in an ensemble of pores with an arbitrary distribution.

The approach has the limitation that the influence of the structure of the matrix on diffusional flow is not explicitely considered so far. Such a generalization can be carried out by applying the results to particular systems of interest.

\section{ACKNOWLEDGMENT}

The present work could be performed due to longstanding support from the Deutsche Forschungsgemeinschaft (DFG) and the Bundesministerium für Bildung, Wissenschaft, Forschung und Technologie (BMBF). One of the authors (J.S.) would like to express, in addition, his gratitude to DFG for a travel grant
(DFG 477/911/96).

\section{REFERENCES}

1. Pascova, R.; Gutzow, I.; Glastechnische Berichte 1983, 56,324

2. Lifshitz, I. M.; Slezov, V. V.; J. Phys. Chem. Solids 1961, 19, 35 and references cited therein.

3. Christian, J. W.; The Theory of Transformations in Metals and Alloys, Oxford 1975.

4. Johnson, W. C.; Howe, J. M.; Laughlin, D. E.; Soffa, W. A. (Eds.): Solid to Solid Phase Transformations, Proceedings of the International Conference on Solid to Solid Phase Transformations in Inorganic Materials PTM94; Nemacolin Woodlands, Farmington, July 1994.

5. Zanotto, E. D.; Müller, E.; J. Non-Crystalline Solids 1991, $130,220$.

6. Schmelzer, J.; Ulbricht, H.; J. Colloid Interface Sci. 1987, 117,325

7. Schmelzer, J.; Thermodynamik finiter Systeme und die Kinetik von thermodynamischen Phasenübergängen 1. Art, Habilarbeit, Rostock 1985.

8. Schmelzer, J.; Gutzow, I.; Z. Phys. Chemie (Leipzig) 1988, 269,753

9. Schmelzer, J.; Gutzow, I.; Pascova, R.; J. Crystal Growth 1990, 104, 505.

10. Schmelzer, J.; Pascova, R.; Gutzow, I.; Phys. Status Solidi 1990, a 117, 363.

11. Gutzow, I.; Schmelzer, J.: The Vitreous State: Thermodynamics, Structure, Rheology, and Crystallization, Springer, Berlin 1995.

12. Kawasaki, K.; Enomoto, Y.; Physica 1988, A 150, 463.

13. Schmelzer, J.; Möller, J.; Phase Transitions 1992, 38, 261.

14. Slezov, V. V.; Schmelzer, J.; Möller, J.; J. Crystal Growth 1993, 132, 419.

15. Schmelzer, J.; Möller, J.; Slezov, V. V.; J. Phys. Chem. Solids 1995, 56, 1013.

16. Slezov, V. V.; Schmelzer, J.; J. Phys. Chem. Solids 1994, $55,243$. 